\title{
Correction to: Non-autoimmune acute-onset type 1 diabetes mellitus newly developed in an elderly patient presenting elevation of serum pancreatic exocrine enzymes
}

\author{
Akinori Kanzaki ${ }^{1,2}$ (1) Morihiro Matsuda ${ }^{1}$ - Shinji Yokota ${ }^{1} \cdot$ Kenji Takai $^{1}$. Jun Murai ${ }^{1}$ - Akihito Otsuka ${ }^{1}$. \\ Hidenori Koyama ${ }^{3} \cdot$ Tadashi Nakamura $^{1}$
}

Published online: 30 September 2021

(c) The Japan Diabetes Society 2021

\section{Correction to: Diabetology International https://doi.org/10.1007/s13340-021-00535-0}

In Table 1 of this article, the value in the row "CMV-IgG" under "Second test" was incorrectly published as 2010 . The correct value is 210 .

The original article was updated.

Publisher's Note Springer Nature remains neutral with regard to jurisdictional claims in published maps and institutional affiliations.

The original article can be found online at https://doi.org/10.1007/ s13340-021-00535-0.

Akinori Kanzaki

aknewendocrine19841223@gmail.com

1 Department of Internal Medicine, Medical Corporation Kawasaki Hospital, 3-3-1 Higashiyama-cho, Kobe, Hyogo 652-0042, Japan

2 Present Address: Department of Internal Medicine, Hyogo College of Medicine, Sasayama Medical Center, 5 Kurooka, Tamba-Sasayama, Hyogo 669-2321, Japan

3 Department of Diabetes, Endocrinology and Clinical Immunology, Hyogo College of Medicine, 1-1 Mukogawa-cho, Nishinomiya, Hyogo 663-8501, Japan 